Article

\title{
Asparagine Metabolism in Tumors Is Linked to Poor Survival in Females with Colorectal Cancer: A Cohort Study
}

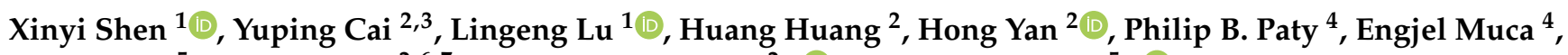 \\ Nita Ahuja ${ }^{5}$, Yawei Zhang ${ }^{2,6,7}$, Caroline H. Johnson ${ }^{2, *}$ (1) and Sajid A. Khan ${ }^{5, *(1)}$
}

check for

updates

Citation: Shen, X.; Cai, Y.; Lu, L.; Huang, H.; Yan, H.; Paty, P.B.; Muca, E.; Ahuja, N.; Zhang, Y.; Johnson, C.H.; et al. Asparagine Metabolism in Tumors Is Linked to Poor Survival in Females with Colorectal Cancer: A Cohort Study. Metabolites 2022, 12, 164. https://doi.org/10.3390/ metabo12020164

Academic Editor: Fidele Tugizimana

Received: 6 January 2022

Accepted: 5 February 2022

Published: 9 February 2022

Publisher's Note: MDPI stays neutral with regard to jurisdictional claims in published maps and institutional affiliations.

Copyright: (C) 2022 by the authors. Licensee MDPI, Basel, Switzerland. This article is an open access article distributed under the terms and conditions of the Creative Commons Attribution (CC BY) license (https:// creativecommons.org/licenses/by/ $4.0 /)$.
1 Department of Chronic Disease Epidemiology, Yale School of Public Health, Yale University, New Haven, CT 06510, USA; xinyi.shen@yale.edu (X.S.); lingeng.lu@yale.edu (L.L.)

2 Department of Environmental Health Sciences, Yale School of Public Health, Yale University, New Haven, CT 06510, USA; yupingcai@sioc.ac.cn (Y.C.); h.huang003@gmail.com (H.H.); hong.yan@yale.edu (H.Y.); zhangyw@cicams.ac.cn (Y.Z.)

3 Interdisciplinary Research Center on Biology and Chemistry, Shanghai Institute of Organic Chemistry, Chinese Academy of Sciences, Shanghai 200032, China

4 Department of Surgery, Memorial Sloan Kettering Cancer Center, New York, NY 10065, USA; patyp@mskcc.org (P.B.P.); mucae@mskcc.org (E.M.)

5 Division of Surgical Oncology, Department of Surgery, Yale University School of Medicine, New Haven, CT 06510, USA; nita.ahuja@yale.edu

6 Department of Surgery, Yale University School of Medicine, New Haven, CT 06510, USA

7 National Cancer Center, National Clinical Research Center for Cancer, Cancer Hospital, Chinese Academy of Medical Sciences and Peking Union Medical College, Beijing 100021, China

* Correspondence: caroline.johnson@yale.edu (C.H.J.); sajid.khan@yale.edu (S.A.K.)

\begin{abstract}
The interplay between the sex-specific differences in tumor metabolome and colorectal cancer (CRC) prognosis has never been studied and represents an opportunity to improve patient outcomes. This study examines the link between tumor metabolome and prognosis by sex for CRC patients. Using untargeted metabolomics analysis, abundances of 91 metabolites were obtained from primary tumor tissues from 197 patients ( $\mathrm{N}=95$ females, $\mathrm{N}=102$ males) after surgical colectomy for stage I-III CRC. Cox Proportional hazard (PH) regression models estimated the associations between tumor metabolome and 5-year overall survival (OS) and recurrence-free survival (RFS), and their interactions with sex. Eleven metabolites had significant sex differences in their associations with 5-year OS, and five metabolites for 5-year RFS. The metabolites asparagine and serine had sex interactions for both OS and RFS. Furthermore, in the asparagine synthetase (ASNS)-catalyzed asparagine synthesis pathway, asparagine was associated with substantially poorer OS (HR (95\% CI): 6.39 (1.78-22.91)) and RFS (HR (95\% CI): 4.36 (1.39-13.68)) for female patients only. Similar prognostic disadvantages in females were seen in lysophospholipid and polyamine synthesis. Unique metabolite profiles indicated that increased asparagine synthesis was associated with poorer prognosis for females only, providing insight into precision medicine for CRC treatment stratified by sex.
\end{abstract}

Keywords: metabolomics; colorectal cancer; prognosis; asparagine metabolism

\section{Introduction}

According to the U.S. Centers for Disease Control and Detection (CDC), colorectal cancer (CRC) was the third leading cause of cancer-related deaths in the United States in 2021 [1]. Prognosis is partly dependent on stage at presentation according to the TNM classification system based on the American Joint Committee on Cancer (AJCC) [2] and the Union for International Cancer Control (UICC) [3]. Additional clinicopathologic variables can also impact survival, such as primary tumor location [4,5], body mass index (BMI) [6], and diabetes conditions [7].

The application of biomarkers in a clinical context has the potential to improve prognosis based on gene mutations [8,9], microRNAs [10], and DNA methylation [11,12]. During 
the last decade, cancer studies have benefited from the implementation of metabolomics [13]. CRC metabolomic studies have shown the correlation of certain metabolite profiles to cancer survival and recurrence using biological samples of urine and tissue [14]. Some plasma metabolites are associated with recurrence in stage II and III patients [15], showing the potential value of metabolomics in finding prognostic biomarkers of CRC.

In addition, sex-related differences in CRC prognosis have become increasingly important in cancer research $[16,17]$. Female patients have a higher prevalence of right-sided colon cancer (RCC), which is associated with poorer overall survival (OS) [18,19]. However, male CRC patients have a survival disadvantage in most subgroups by anatomic location and age [20]. At present, female and male patients do not receive different sex-specific therapies.

Previously, our untargeted metabolomics analyses in a large series of CRC tumor tissues revealed sex-specific metabolic subphenotypes that could influence tumor progression and response to therapeutics [21]. Important metabolic pathways in these subphenotypes included asparagine synthesis, energy production (glycolysis and pentose phosphate pathway (PPP)), lysophospholipid synthesis, methionine metabolism, and polyamine synthesis [21]. Particularly, female patients with RCC showed increased asparagine levels [21], which can be produced intracellularly by an ATP- and glutamine-dependent reaction catalyzed by asparagine synthetase (ASNS) [22]. By examining The Cancer Genome Atlas (TCGA) data, we discovered that high ASNS expression was associated with poorer OS for female CRC patients [21]. Additionally, increased ASNS expression has been linked to aggressive tumors and poorer prognosis in other cancer types $[23,24]$. These findings suggest that sex plays a vital role in CRC prognosis together with the covariates of metabolism and anatomic tumor location. A clear understanding of these sex-specific metabolic differences represents a golden opportunity to prolong the survival of CRC patients.

In this study, we examine whether there is a link between CRC tumor metabolites and prognosis stratified by sex of the patient. This is the first study to reveal that sex-related differences exist in primary tumor metabolite profiles that are associated with OS and recurrence-free survival (RFS) in patients who have undergone curative intent colectomy. We also identify metabolic pathways which are associated with poor survival only in female CRC.

\section{Results}

\subsection{Population Characteristics}

Baseline characteristics of 197 patients ( $\mathrm{N}=102$ males, $\mathrm{N}=95$ females), including 5-year OS and 5-year RFS, are provided in Table 1. Median follow-up time since date of surgery was 74.8 months (0.1-169.2). Older age, administration of chemotherapy, and advanced clinical stage were inversely related to OS (Table 1). Each subgroup by anatomic tumor location and clinical stage, pathological information (KRAS mutation, BRAF mutation and microsatellite instability status), demographic characteristics, including race/ethnicity, are displayed in Table S1. Prognosis among different subgroups is shown in Table S2.

Table 1. Demographic Characteristics and Clinical Factors.

\begin{tabular}{|c|c|c|c|c|c|c|c|c|}
\hline \multicolumn{2}{|c|}{ Characteristics } & \multirow{2}{*}{ No. of Patients } & \multicolumn{3}{|c|}{ 5-Year OS } & \multicolumn{3}{|c|}{ 5-Year RFS } \\
\hline & & & Deaths, No. & Rate, $\%{ }^{a}$ & $p^{\mathrm{b}}$ & Cases, No. & Rate, $\%{ }^{a}$ & $p^{\mathrm{b}}$ \\
\hline \multirow{4}{*}{ Age at diagnosis, $\mathrm{y}$} & $\leq 60$ & 19 & 2 & 88.9 & \multirow{4}{*}{0.048} & 3 & 84.2 & \multirow{4}{*}{0.21} \\
\hline & $61-69$ & 64 & 9 & 83.4 & & 14 & 72.4 & \\
\hline & $70-79$ & 81 & 15 & 78.7 & & 12 & 81.4 & \\
\hline & $\geq 80$ & 33 & 11 & 61.5 & & 1 & 96.3 & \\
\hline \multirow{2}{*}{ Sex, $n$} & Male & 102 & 23 & 74.3 & \multirow{2}{*}{0.18} & 17 & 77.5 & \multirow{2}{*}{0.48} \\
\hline & Female & 95 & 14 & 83.2 & & 13 & 84.0 & \\
\hline
\end{tabular}


Table 1. Cont.

\begin{tabular}{|c|c|c|c|c|c|c|c|c|}
\hline \multirow{2}{*}{\multicolumn{2}{|c|}{ Characteristics }} & \multirow{2}{*}{ No. of Patients } & \multicolumn{3}{|c|}{ 5-Year OS } & \multicolumn{3}{|c|}{ 5-Year RFS } \\
\hline & & & Deaths, No. & Rate, $\%$ a & $p^{\mathrm{b}}$ & Cases, No. & Rate, $\%$ a & $p^{\mathrm{b}}$ \\
\hline \multirow{3}{*}{ Clinical stage, n } & $\mathrm{I}$ & 47 & 3 & 92.5 & \multirow{3}{*}{0.001} & 5 & 88.4 & \multirow{3}{*}{0.09} \\
\hline & II & 86 & 13 & 82.4 & & 11 & 82.0 & \\
\hline & III & 64 & 21 & 63.5 & & 14 & 73.8 & \\
\hline \multirow{2}{*}{ Chemotherapy, $\mathrm{n}$} & Yes & 66 & 18 & 68.5 & \multirow{2}{*}{0.03} & 15 & 73.1 & \multirow{2}{*}{0.03} \\
\hline & No & 131 & 19 & 83.7 & & 15 & 85.0 & \\
\hline \multirow{2}{*}{$\begin{array}{l}\text { Anatomic tumor } \\
\text { location, } \mathrm{n}\end{array}$} & Left & 99 & 17 & 81.2 & \multirow{2}{*}{0.42} & 19 & 77.2 & \multirow{2}{*}{0.23} \\
\hline & Right & 98 & 20 & 75.6 & & 11 & 85.3 & \\
\hline
\end{tabular}

a Survival rates were calculated by the Kaplan-Meier estimation method. ${ }^{b} p$ value of Log-rank test.

\subsection{Sex-Specific Differences in the Associations between Individual Metabolite Abundances and CRC Prognosis}

A total of 18 metabolites were significantly associated with OS in males or females or in both sexes (Table S3), adjusted for anatomic location, clinical stage, chemotherapy history, and age, similarly, 25 metabolites had significant correlations with RFS (Table S4). Only carnitine and hypoxanthine remained significantly associated with RFS for males after false discovery rate (FDR) adjustment.

Multivariate Cox $\mathrm{PH}$ models on single metabolites and their associations with OS or RFS revealed dichotomous findings by sex. Figure 1 presents the metabolites (adenosine, asparagine, citrulline, glycerol 3-phosphate (Gro3P), lysophosphatidylcholine (LysoPC)(16:0), ornithine, succinate, threonine, UDP-D-glucose, uracil, and xanthosine) that had sex differences in their associations with CRC prognosis $\left(P_{\text {interaction }}<0.05\right)$. Among these 11 metabolites, succinate was significantly associated with improved OS for female patients $\left(\mathrm{HR}_{\mathrm{OS}}=0.35,95 \% \mathrm{CI}=0.12-0.99 ; p=0.047\right)$, while it was associated with poorer $\mathrm{OS}$ for males $\left(\mathrm{HR}_{\mathrm{OS}}=1.91,95 \% \mathrm{CI}=1.23-2.96, p=0.004\right)$ (Figure $1 \mathrm{~A}$ ). Five metabolites (argininosuccinic acid, asparagine, creatinine, hypoxanthine, and serine) showed significance for RFS and sex-specific differences (Figure 1B). Of these metabolites, asparagine exhibited sex differences for both OS and RFS ( $\left.P_{\text {interaction, OS }}=0.03, P_{\text {interaction, RFS }}=0.007\right)$, and similarly for serine $\left(P_{\text {interaction, OS }}=0.04, P_{\text {interaction,RFS }}=0.01\right)($ Tables S3 and S4). Asparagine was significantly associated with better CRC prognosis in males $\left(\mathrm{HR}_{\mathrm{OS}}=0.72,95 \% \mathrm{CI}=0.54-0.96\right.$, $\left.p=0.03 ; \mathrm{HR}_{\mathrm{RFS}}=0.74,95 \% \mathrm{CI}=0.56-0.97, p=0.03\right)$, while its associations with worse prognosis in females trended towards significance $\left(P_{\mathrm{OS}}=0.13, P_{\mathrm{RFS}}=0.09\right.$, Tables S3 and S4). Similarly, serine was significantly associated with better prognosis in males $\left(\mathrm{HR}_{\mathrm{OS}}=0.55\right.$, $95 \% \mathrm{CI}=0.37-0.81, p=0.002$. $\left.\mathrm{HR}_{\mathrm{RFS}}=0.57,95 \% \mathrm{CI}=0.40-0.83, p=0.003\right)$, while it has no significant associations with prognosis in females (Tables S3 and S4).

A

\section{Females (OS)}

Metabolite

Adenosine Asparagine Citrulline

Glycerol 3-phosphate $3.64(1.26-10.49)$

LysoPC(16:0) $\quad 1.52(1.03-2.25)$ Serine

Succinate

Threonine UDP-D-Glucose Uracil Xanthosine
$\operatorname{HR}(95 \% \mathrm{Cl})$

$0.90(0.69-1.17)$

$1.51(0.89-2.57)$ $1.66(0.97-2.83)$

$1.25(0.65-2.41)$

$0.35(0.12-0.99)$

$1.12(0.67-1.87)$

$0.81(0.67-0.97)$

$1.24(0.56-2.76)$

$1.22(0.86-1.73)$

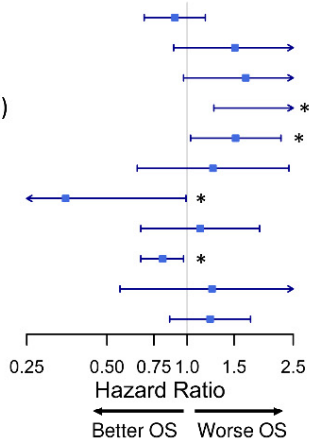

Males (OS)

HR $(95 \%$ Cl)

$1.29(1.03-1.62)$

$0.72(0.54-0.96)$

$0.65(0.46-0.92)$

$0.91(0.47-1.77)$

$0.85(0.65-1.11)$

$0.55(0.37-0.81)$

$1.91(1.23-2.96)$

$0.61(0.44-0.85)$

$1.16(0.95-1.41)$

$0.42(0.26-0.69)$

$0.71(0.54-0.94)$

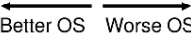

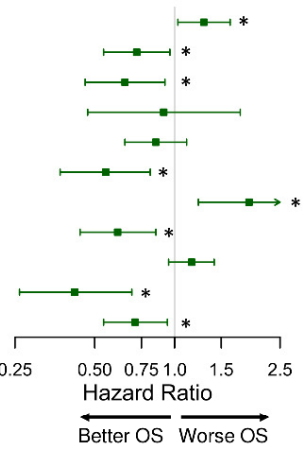

Figure 1. Cont. 
B

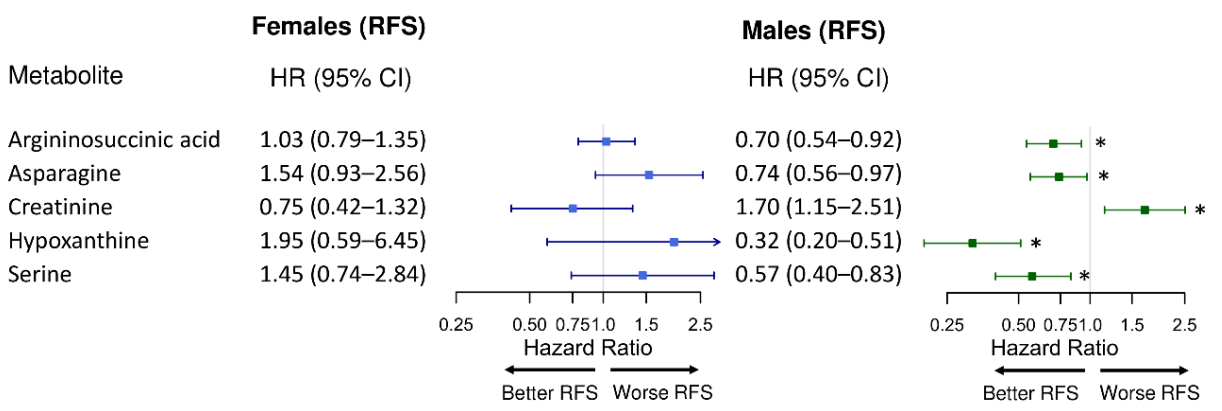

Figure 1. Sex-specific differences in the associations between individual metabolite and CRC prognosis. (A) 5-year overall survival (OS) and (B) 5-year recurrence-free survival (RFS). LysoPC: lysophosphatidylcholine. All metabolites were observed with significant sex interactions $\left(P_{\text {interaction }}<0.05\right)$. Hazard ratio (HR) for each metabolite ( $\log _{2}$-transformed abundance) was calculated by multivariate Cox $\mathrm{PH}$ regression adjusted for anatomic location, chemotherapy history, clinical stage, and age (continuous). Error bars represent 95\% confidence intervals (CIs). The 95\% CIs marked with asterisks indicate significant associations between the metabolite and the corresponding prognosis ( $p$ values $<0.05$ ): A metabolite with $\mathrm{HR}<1$ was associated with better prognosis; a metabolite with HR $>1$ was associated with worse prognosis. The x-axes are log-scaled.

Additional analysis using cases with complete information of $K R A S$ mutation, $B R A F$ mutation and microsatellite instability status showed that 15 metabolites were associated with OS and 3 metabolites were associated with RFS with significant $P_{\text {interaction }}$ (Appendix B, Tables A1 and A2). Asparagine was associated with poor RFS in females but not males with a $P_{\text {interaction,RFS }}$ close to significance (Appendix B, Table A2).

\subsection{Sex-Specific Differences in CRC Prognosis Associated with Asparagine Synthesis}

We examined sex-specific associations between the ASNS-catalyzed asparagine synthesis pathway and CRC prognosis (Tables S5 and S6). Significant interactions with sex were detected in associations between asparagine abundance and both OS and RFS $\left(P_{\text {interaction, OS }}=0.02, P_{\text {interaction, RFS }}=0.003\right)$ as summarized as Model 1 , where asparagine abundance was treated as a continuous variable (Table 2). In Model 1, asparagine was significantly associated with poor $\mathrm{OS}\left(\mathrm{HR}_{\mathrm{OS}}=6.39,95 \% \mathrm{CI}=1.78-22.91, p=0.004\right)$ and poor RFS $\left(\mathrm{HR}_{\mathrm{RFS}}=4.36,95 \% \mathrm{CI}=1.39-13.68, p=0.01\right)$ only in females, while asparagine was only significantly associated with better OS only in males $\left(\mathrm{HR}_{\mathrm{OS}}=0.57,95 \% \mathrm{CI}=0.36-0.91\right.$, $p=0.02$ ) after adjustment for clinical stages, chemotherapy history, age, anatomic location, and other crucial metabolites involved in ASNS expression (Table 2).

Table 2. Multivariate analysis of asparagine synthesis catalyzed by asparagine synthetase (ASNS).

\begin{tabular}{|c|c|c|c|c|c|c|c|}
\hline \multirow{2}{*}{ Prognosis } & \multirow{2}{*}{ Sex } & \multicolumn{3}{|c|}{ Model 1 a } & \multicolumn{3}{|c|}{ Model $2^{b}$} \\
\hline & & $\operatorname{HR}(95 \% \mathrm{CI})^{\mathrm{c}}$ & $p$ & $p_{\text {interaction }} \mathrm{d}$ & $\operatorname{HR}(95 \% \mathrm{CI})^{\mathrm{c}}$ & $p$ & $p_{\text {interaction }}{ }^{\mathrm{d}}$ \\
\hline \multirow{2}{*}{ OS } & Females & 6.39 (1.78-22.91) & 0.004 & \multirow{2}{*}{0.02} & $5.68(1.06-30.61)$ & 0.04 & \multirow{2}{*}{0.052} \\
\hline & Males & $0.57(0.36-0.91)$ & 0.02 & & $0.46(0.11-1.84)$ & 0.27 & \\
\hline \multirow{2}{*}{ RFS } & Females & $4.36(1.39-3.68)$ & 0.01 & \multirow{2}{*}{0.003} & 4.89 (1.07-22.39) & 0.04 & \multirow{2}{*}{0.03} \\
\hline & Males & $0.96(0.61-1.50)$ & 0.86 & & $1.15(0.27-4.80)$ & 0.85 & \\
\hline
\end{tabular}

a Asparagine abundance was considered as a continuous variable ( $\log _{2}$ transformed). ${ }^{\mathrm{b}}$ Asparagine abundance was dichotomized to low levels ( $\leq 75 \%$ percentile of asparagine abundance among all patients) (reference) and high levels ( $>75 \%$ percentile). ${ }^{c}$ Hazard ratio for asparagine abundance in the corresponding multivariate Cox $\mathrm{PH}$ model, adjusted for anatomic location, clinical stages, chemotherapy history, age (continuous), and other crucial metabolites involved in ASNS-catalyzed asparagine synthesis (aspartate, glutamate, glutamine, and AMP were treated as continuous variables and were $\log _{2}$ transformed). ${ }^{\mathrm{d}} p$ values for the interaction between sex and asparagine. 
An additional multivariate Cox $\mathrm{PH}$ regression model was built; Model 2, where asparagine abundance was dichotomized to low levels and high levels. There was a significant interaction between sex and dichotomized asparagine levels for RFS and a similar interaction that was approaching significance for OS (Table 2) $\left(P_{\text {interaction,OS }}=0.052\right.$, $P_{\text {interaction, } \mathrm{RFS}}=0.03$ ).

Figure 2 displays the Cox adjusted survival curves for dichotomized asparagine levels based on Model 2, illustrating that female CRC patients with a high level of ASNS-catalyzed asparagine had significantly poorer OS (Figure 2A) and poorer RFS (Figure 2C) compared with female patients with a low level. Strikingly, levels of asparagine were not associated with OS (Figure 2B) or RFS (Figure 2D) for male CRC patients.

A
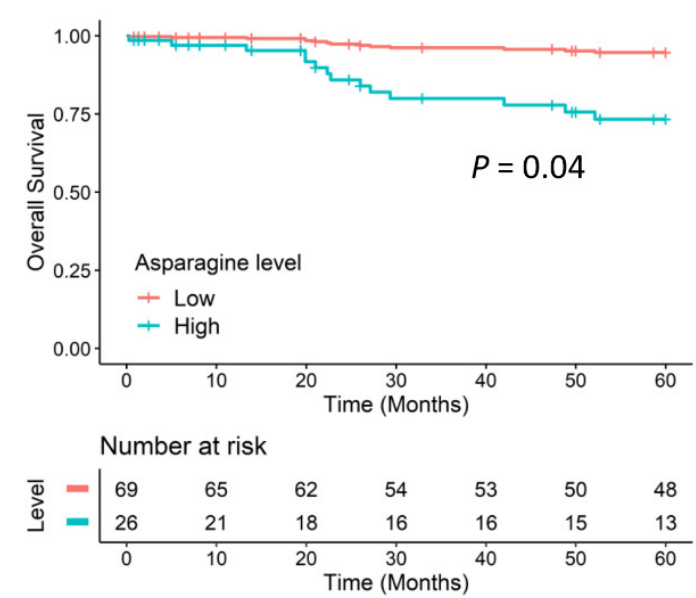

C
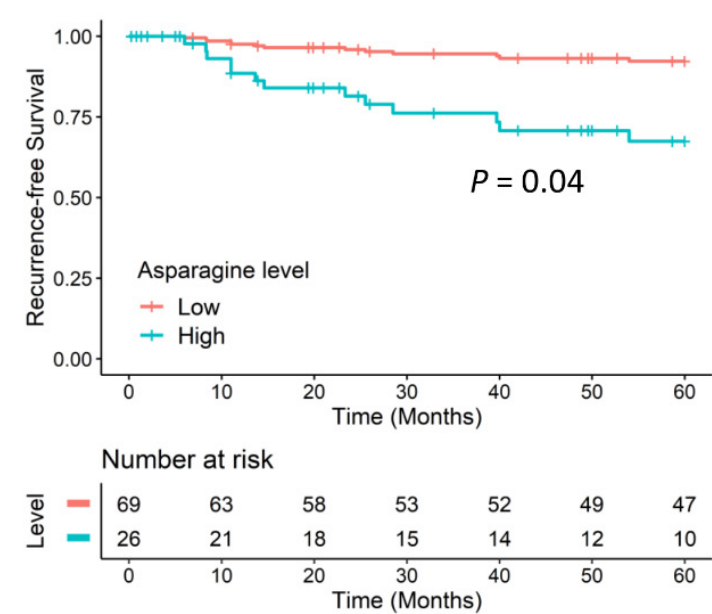

B

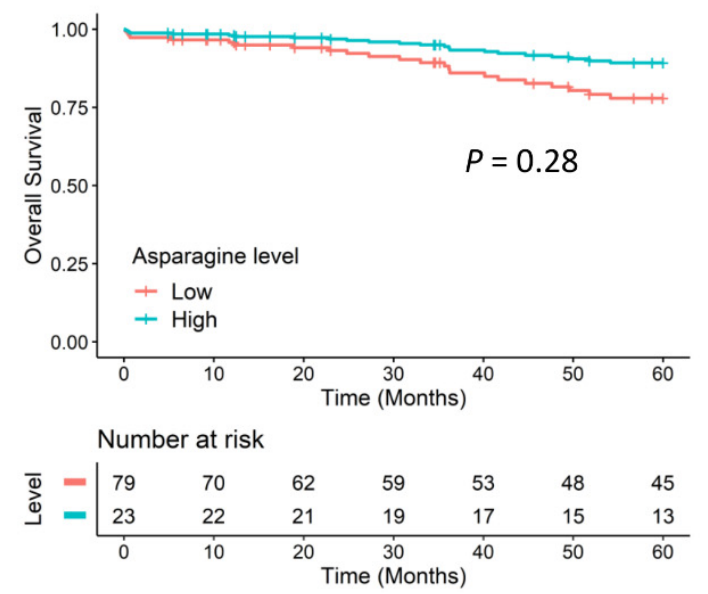

D

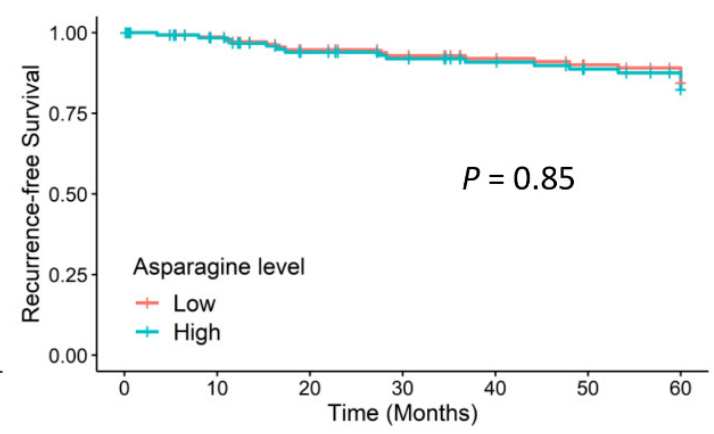

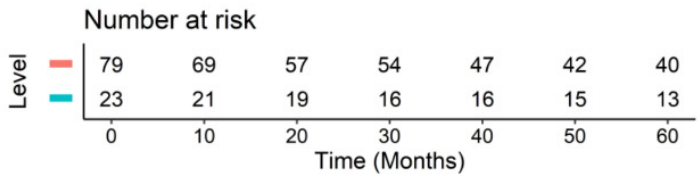

Figure 2. Cox adjusted survival curves of ASNS-catalyzed asparagine. (A) OS of females, (B) OS of males, (C) RFS of females, and (D) RFS of males. Results were based on Model 2 (Table 2) using multivariate Cox $\mathrm{PH}$ regression, adjusted for anatomic location, chemotherapy history, clinical stages, age (continuous), and other crucial metabolites involved in ASNS-catalyzed asparagine synthesis (aspartate, glutamate, glutamine, and AMP were treated as continuous variables and were $\log _{2}$ transformed). $p$ values for the associations between dichotomized asparagine levels and the prognosis in Model 2.

ASNS is induced by the PI3K-AKT-mTOR pathway and mutated KRAS [21]. Within our study cohort, we had information of KRAS status for 161 patients by KRAS status. Therefore, we performed a further analysis similar to Model 1 on this subset of patients 
(Appendix B, Tables A3 and A4), and asparagine still showed similar sex-specific associations with both OS and RFS.

\subsection{Sex-Specific Differences in CRC Prognosis Associated with Glycolysis and the Pentose Phosphate Pathway}

Considering glycolysis and the PPP metabolism, the association between Gro3P and OS for females trended towards significance $(p=0.08)$, and there was no association in males $(p=0.76)$ (Table S8). Gro3P was significantly associated with a lower risk of 5-year recurrence only for males $\left(\mathrm{HR}_{\mathrm{RFS}}=0.27,95 \% \mathrm{CI}=0.08-0.88, p=0.03\right)$, though no sex differences were detected $\left(p_{\text {interaction }}=0.25\right)$ (Tables S7 and S8).

\subsection{Sex-Specific Differences in CRC Prognosis Associated with Lysophospholipid Synthesis}

We analyzed the relationships between CRC prognosis and important metabolites involved in lysophospholipid synthesis. The interactions between sex and LysoPC (16:0), LysoPC (18:1), lysophosphatidylethanolamine (LysoPE) (18:0), and LysoPE (22:5) in the association with OS were significant (Table S9). In the multivariate analysis on OS for lysophospholipid synthesis by sex, LysoPC (16:0) was associated with poor OS only for females $\left(\mathrm{HR}_{\mathrm{OS}}=2.07,95 \% \mathrm{CI}=1.06-4.07, p=0.03\right)$, while it was not significantly associated with OS for males $(p=0.32)$ (Table S10). In the models of RFS for lysophospholipid synthesis by sex, LysoPE (22:5) presented a robust trend toward a significant sex difference in its association with RFS ( $P_{\text {interaction }}=0.052$, Table S9). LysoPE (22:5) was associated with increased risks of 5-year recurrence of CRC for females ( $\mathrm{HR}_{\mathrm{RFS}}=5.38,95 \% \mathrm{CI}=1.02-28.42$, $p=0.048)$, while it was associated with reduced risk of recurrence for males $\left(\mathrm{HR}_{\mathrm{RFS}}=0.10\right.$, $95 \%$ CI $=0.02-0.62, p=0.01$ ) (Table S10).

\subsection{Sex-Specific Differences in CRC Prognosis Associated with Methionine Metabolism}

Analysis of methionine metabolism for all patients indicated that serine favored better OS $\left(\mathrm{HR}_{\mathrm{OS}}=0.56,95 \% \mathrm{CI}=0.34-0.92, p=0.02\right)$ and $\mathrm{RFS}\left(\mathrm{HR}_{\mathrm{RFS}}=0.49,95 \% \mathrm{CI}=0.29-0.83\right.$, $p=0.008$ ) (Table S11). After further stratification by sex, serine was associated with better OS in males $\left(\mathrm{HR}_{\mathrm{RFS}}=0.37,95 \% \mathrm{CI}=0.19-0.72, p=0.004\right)$ and improved RFS in males as well $\left(\mathrm{HR}_{\mathrm{RFS}}=0.49,95 \% \mathrm{CI}=0.24-0.98, p=0.04\right)$, while its associations with $\mathrm{CRC}$ prognosis in females were not significant (Table S12). The interaction between serine and sex in terms of OS and RFS were both significant $\left(P_{\text {interaction, OS }}=0.04, P_{\text {interaction, }, \mathrm{RFS}}=0.045\right.$, Table S11) . Methionine had a significant sex interaction (Table S11), but it was not significantly associated with OS or RFS in either sex (Table S12). The additional metabolites in this pathway did not have significant sex interactions (Table S11).

\subsection{Sex-Specific Differences in CRC Prognosis Associated with Polyamine Synthesis}

Lastly, we examined metabolites involved in polyamine synthesis. Arginine had a marked trend towards a significant sex interaction with OS $\left(p_{\text {interaction }}=0.07\right)$, and a significant sex interaction for RFS ( $\left.p_{\text {interaction }}=0.04\right)($ Table S13). Further stratified by sex, arginine was associated with worse OS only for females $\left(\mathrm{HR}_{\mathrm{OS}}=5.63,95 \% \mathrm{CI}=1.33-23.78\right.$, $p=0.02)$, while its association with OS in males was not significant $(p=0.87)$ (Table S14). For RFS, arginine was associated with higher risks of 5 -year CRC recurrence only for females $\left(\mathrm{HR}_{\mathrm{RFS}}=3.22,95 \% \mathrm{CI}=1.14-9.13, p=0.03\right)$ while it had no significant association with RFS for males $(p=0.24)$ (Table S14). Citrulline suggested significant sex interactions with both OS and RFS ( $\left.p_{\text {interaction,OS }}=0.002, p_{\text {interaction,RFS }}=0.02\right)($ Table S13): it had a significant association with poorer OS for females $\left(\mathrm{HR}_{\mathrm{OS}}=2.57,95 \% \mathrm{CI}=1.04-6.33, p=0.04\right)$ but not for males $(p=0.18)$, while it was not significantly associated with RFS in either sex (Table S14). Other critical metabolites in polyamine synthesis did not have significant sex interactions (Table S13). 


\section{Discussion}

To our knowledge, this is the first study to show that sex-specific differences in the CRC tumor metabolome are linked to prognosis based on data from one of the largest metabolomics study cohorts. It is also the first application of a novel approach to examine metabolic pathways and their associations to CRC prognosis. By applying state-of-the-art mass spectrometry to fresh frozen specimens acquired operatively in a large colorectal biorepository, we show that females have a distinct metabolite profile, underscored by asparagine metabolism, which correlates with prognosis after surgical colectomy. This suggests that females and males have unique colorectal tumor metabolism, which contributes to sex-specific clinical outcomes.

Our study revealed that 11 tumor metabolites were associated with CRC 5-year OS and five metabolites for 5-year RFS, and these associations were sex-specific. Asparagine and serine were associated with both 5-year OS and 5-year RFS in a sex-specific manner. Further examination of asparagine within the de novo asparagine synthesis pathway (catalyzed by ASNS), showed consistently poor OS and RFS with high asparagine abundance for females. This supports previous findings revealing poor female survival and high ASNS expression from the TCGA [21]. In addition, we previously hypothesized that asparagine is linked to tumor aggressiveness in females with RCCs as it correlates with increased threonine and serine uptake, which indicates aggressive tumor phenotypes [21]. ASNS-catalyzed asparagine production has also been shown to be crucial for in vitro cancer cell proliferation by amino acid homeostasis [25], and the availability of asparagine in vitro and in vivo propagates the metastatic progression of breast cancer [26].

In CRC, SRY-box 12 (SOX12) expression promotes cell proliferation and metastasis, and it facilitates ASNS expression [27]. In addition, expression of the mutant KRAS gene correlates with a marked decrease in aspartate levels and increased asparagine levels due to upregulated ASNS expression, which indicated that ASNS might be a novel therapeutic target for KRAS-mutant CRC [28,29]. Moreover, SLC25A22 serves as an essential metabolic regulator for $C R C$ progression [30] by promoting the synthesis of aspartate-derived amino acids (asparagine) in KRAS-mutant CRC cells [31]. In our study, KRAS information was incomplete but further analysis on patients with complete $K R A S$ records can be found in Appendix B (Tables A1 and A2), where asparagine showed similar sex-specific associations with both OS and RFS. Furthermore, increased dietary asparagine in animals promotes metastatic progression in breast cancer, and L-asparaginase treatment or dietary asparagine restriction inhibits metastasis [32]. Hence, both internally produced asparagine and external exposure to asparagine through diet may influence CRC tumor progression. However, a review study pointed out that some studies are contradictory regarding whether ASNS expression favors or inhibits CRC progression [33], while our findings indicate that such inconsistency may attribute to sex differences.

As mentioned, serine was associated with both 5-year OS and RFS in a sex-specific manner. Our data showed that serine favored longer OS and RFS in males but was independent of prognosis in females. Serine metabolism has been reported to contribute to CRC metabolism and growth [34] and enzymes involved in serine synthesis such as phosphoglycerate dehydrogenase (PHGDH) [35] are found in higher levels in colonic tumor tissue than paired normal tissue [36]. These studies did not consider sex differences that might lend to a potential sex-specific phenomenon.

Our results indicated that sex significantly modified the associations between lysophospholipid synthesis and CRC prognosis. LysoPC (16:0) was associated with worse OS for females in both individual metabolite analysis and the model for lysophospholipid synthesis. Our previous study identified that multiple LysoPCs and LysoPEs were upregulated in female patients with RCC (stage I) only, suggesting that the higher level of lysophospholipids in women with RCC would promote fatty acid supply that is essential for cancer cell growth at early stages [21]. It has also been discussed that sex-specific estrogen regulation may inhibit CRC cell survival by suppressing triglyceride biosynthesis, a vital lipid marker related to CRC progression [17]. 
Lastly, sex interacts with the associations between polyamine metabolism and CRC prognosis. Arginine and citrulline were associated with worse RFS and OS, respectively, for females but not for males. A previous study investigated the L-arginine/nitric oxide (NO) pathway in CRC by clinical stage, location of a primary tumor, and sex, and they observed elevated arginine levels in LCCs compared with RCCs, and a drop in arginine levels in the early postoperative period in females exclusively [37]. Arginine and citrulline are important parts of the urea cycle [38], where ornithine is produced and metabolized into polyamines by ornithine decarboxylase (ODC) [39]. ODC is a target for inhibition by difluoromethylornithine (DFMO) (an anti-polyamine therapy for colorectal neoplasia) [40], therefore, some of the polyamine precursors could be important for polyamine treatment responses, where sex can play a role. Moreover, there are NCI-funded cooperative group CRC chemoprevention trials targeting polyamine synthesis (PACES/SWOG) [41], and a review study showed that methionine dietary restriction can suppress tumor formation, potentially through reducing the availability of decarboxylated SAM which is a cofactor for spermidine (a polyamine) production [42]. Therefore, key metabolic pathways may have great applicability in sex-specific treatment or preventative interventions for CRC.

Although all female patients in our analysis were over 55 years and sex hormones might not be able to alter the associations between tumor metabolome and CRC prognosis, the observed sex differences could be attributed to other factors. Sex differences in metabolism are known to exist throughout life-course, as reflected by changes in amino acids level at birth [43], fluctuation of lipid profile of males before and after puberty, with larger sex differences at older ages [44]. Sex chromosomes such as the gene forkhead box P3 (FOXP3) located on the X chromosome have been linked to CRC [45]. In addition, there is evidence that sex differences exist before puberty when sex hormone levels rise, and life-course exposure to estradiol can result in elevated DNA and CpG island methylation, as well as demethylation which could result in genetic mutations at later life [46]. Life-course exposure to sex hormones can alter microbiome diversity which would have an impact on microbial metabolism in the colon [47]. Sex differences in the tumor microenvironment such as hypoxia, drug metabolism, and angiogenesis have been shown to impact sex-specific immune responses and oncogenes in CRC [17].

Some of the study limitations include the number of events in the cohorts compared to sample size, and the multiple categories such as clinical stage, sex group, anatomic location, which might weaken statistical power. In addition, prognostic factors such as KRAS [48] and $B R A F$ mutational status [49], and microsatellite instability status [50] were incomplete in our data; thus, they were not considered in survival analyses for metabolic pathways to avoid less statistical power. Therefore, future studies from an independent cohort with more comprehensive molecular profiling will be valuable. Further, metabolomic analysis of normal colorectal tissues that surround the tumor would also enhance the current findings.

In conclusion, we apply a novel methodology using tumor tissue to identify sexspecific metabolic pathways associated with CRC prognosis in a surgical cohort. We show that tumor metabolite levels have different associations with patient prognosis by sex, in particular ASNS-catalyzed asparagine production that is linked to poor prognosis only in females. Our study shows that sex-specific tumor metabolism is an important factor for understanding differences in patient prognosis and potentially differences in treatment responses. Accordingly, we recommend that the design of precision medicine approaches consider both tumor metabolism and sex to obtain more robust findings in clinical trials and improve patient outcomes.

\section{Materials and Methods}

\subsection{Sample Collection and Metabolite Measurements}

Fresh frozen tumor tissue samples were acquired after curative intent colectomy for patients ( $\mathrm{N}=197$ ) with RCCs (cecum, ascending colon, and hepatic flexure) or LCCs (splenic flexure, descending colon, sigmoid, rectosigmoid) with stage I-III CRC (1991-2000). No patients received neoadjuvant chemotherapy. Rectal cases had different biology mechanisms 
to the colon, and transverse tissue could not be accurately assigned as right or left in original, and thus they were not included. Stage IV tissues would have been treated with chemotherapy before surgery, which were therefore excluded. Finally, abundances of 91 metabolites were obtained as published previously [21]. Metabolites were extracted and analyzed by hydrophilic interaction liquid chromatography mass spectrometry (HILIC-MS) and reverse phase liquid chromatography mass spectrometry (RPLC-MS)-based metabolomics using a UPLC system (H-Class ACQUITY, Waters Corporation, Massachusetts, United States) with a quadrupole time-of flight (QTOF) mass spectrometer (Xevo G2-XS QTOF, Waters Corporation, Milford, Massachusetts, United States) as previously described [21]. Other pathological clinical records such as KRAS mutation, BRAF mutation and microsatellite instability status were available but incomplete. More information about and explanations the timeline of sample collection, and metabolite measurements are presented in Appendix A. Yale University IRB determined that this study was not considered Human Subjects Research and did not require IRB review (IRB/HSC no. 1612018746).

\subsection{Statistical Analysis}

We included age, sex, anatomic primary tumor location, chemotherapy history, and clinical stage as covariates. Missing values of metabolites abundances were replaced using median imputation. Multivariable Cox proportional hazard (Cox $\mathrm{PH}$ ) regression models were constructed to evaluate the associations between prognosis with individual metabolite abundance per standard deviation (SD) increase on a $\log _{2}$-scale, adjusted for covariates in either all patients or stratified by sex. Assessment of associations between sex-specific metabolic pathways and prognosis was conducted by multivariate Cox $\mathrm{PH}$ models that included important metabolites involved in these pathways. Considering statistical power, cases with complete pathological information (KRAS mutation, BRAF mutation and microsatellite instability status) were only sufficient to analyze associations between prognosis and individual metabolites but were not to analyze metabolic pathways. Sex-specific difference was examined in multivariate Cox PH models by including an interaction between sex and the metabolite. The 5-year OS and 5-year RFS were calculated. Due to the absence of death events in female patients at clinical stage I, we recoded the clinical stages I and II as "early stage", and III as "late stage". Patients with adjuvant postoperative chemotherapy were coded as having chemotherapy history. Survival analyses were conducted using package "survival" in R (version 4.0.4). A two-sided $p$ value less than .05 was considered statistically significant.

Supplementary Materials: The following supporting information can be downloaded at: https: //www.mdpi.com/article/10.3390/metabo12020164/s1, Table S1: Demographic characteristics and clinical factors for each subgroup, Table S2: Prognosis of patients in early stage (Stage I and II) vs. late stage (Stage III), Table S3: Multivariate analyses of the associations between individual metabolites and 5-year OS by sex, adjusted for anatomic location, chemotherapy history, clinical stages, and age, Table S4: Multivariate analyses of the associations between individual metabolites and 5-year RFS by sex, adjusted for anatomic location, chemotherapy history, clinical stages, and age, Table S5: Multivariate analysis of association between metabolites in ASNS-catalyzed asparagine synthesis pathway and OS and RFS for all patients, Table S6: Multivariate analysis of association between metabolites in ASNS-catalyzed asparagine synthesis metabolic pathway and OS and RFS by sex, Table S7: Multivariate analysis of association between metabolites in PPP and glycolysis metabolic pathway and OS and RFS for all patients, Table S8: Multivariate analysis of association between metabolites in PPP and glycolysis metabolic pathway and OS and RFS by sex, Table S9: Multivariate analysis of association between metabolites in lysophospholipids synthesis and OS and RFS for all patients, Table S10: Multivariate analysis of association between metabolites in lysophospholipids synthesis and OS and RFS by sex, Table S11: Multivariate analysis of association between metabolites in methionine metabolism and OS and RFS for all patients, Table S12: Multivariate analysis of association between metabolites in methionine metabolism and OS and RFS by sex, Table S13: Multivariate analysis of association between metabolites in polyamine synthesis and OS and RFS for all patients, 
Table S14: Multivariate analysis of association between metabolites in polyamine synthesis and OS and RFS by sex.

Author Contributions: Conceptualization, C.H.J., S.A.K., Y.C., X.S., L.L. and Y.Z.; data curation, Y.C., P.B.P., E.M., C.H.J. and S.A.K.; formal analysis, X.S. and Y.C.; software, X.S. and Y.C.; funding acquisition, C.H.J. and S.A.K.; Investigation, Y.C., P.B.P., E.M., C.H.J. and S.A.K.; Methodology, X.S., Y.C., L.L., H.H., and Y.Z.; project administration, C.H.J. and S.A.K.; resources, P.B.P., E.M., C.H.J. and S.A.K.; supervision, C.H.J. and S.A.K.: visualization, X.S.; writing-original draft, X.S.; writing-review and editing, X.S., Y.C., L.L., H.H., H.Y., P.B.P., E.M., N.A., Y.Z., C.H.J. and S.A.K. All authors have read and agreed to the published version of the manuscript.

Funding: This research was funded by NIH 1R21CA223686-01 (C.H.J., S.A.K.) and American Cancer Society research scholar grant 134273-RSG-20-065-01-TBE (C.H.J.). This publication was also made possible by CTSA grant number UL1 TR001863 from the National Center for Advancing Translational Science (NCATS), components of the National Institutes of Health (NIH), and NIH roadmap for Medical Research. Its contents are solely the responsibility of the authors and do not necessarily represent the official view of NIH. This work was also supported by the Lampman Research Fund in Yale Surgical Oncology.

Institutional Review Board Statement: Yale University IRB determined that this study was not considered 'Human Subjects Research and did not require IRB review (IRB/HSC\# 1612018746).

Informed Consent Statement: Not applicable.

Data Availability Statement: The untargeted metabolomics data underlying this article are available at www.ebi.ac.uk/metabolights (accessed on 23 January 2022) MTBLS1122, MTBLS1124, MTBLS1129, MTBLS1130.

Acknowledgments: C.H.J. was supported by the National Cancer Institute of the National Institutes of Health under award number K12CA215110. The content is solely the responsibility of the authors and does not necessarily represent the official views of the National Institutes of Health.

Conflicts of Interest: N.A. received grant funding from Cepheid, Astex, and the National Cancer Institute of the National Institutes of Health. N.A. has licensed methylation biomarkers to Cepheid.

\section{Appendix A}

In 2020, the clinical data on patient outcomes was retrieved from medical records held by Memorial Sloan Kettering Cancer Center (MSKCC). All patients were followed from their operation date for follow up. The HILIC-MS analysis was performed from 31 December, 2018, through 4 January, 2019, and the RPLC-MS was performed from 9 January through 18 January, 2019. Data analysis was conducted from 25 March, 2020, through 13 September, 2021.

The tissue samples were stored at $-80^{\circ} \mathrm{C}$, properly, with minimum degradation, which allowed us to perform metabolomics recently and, thus, find associations between metabolites and prognostic outcomes. The patients were not treated with chemotherapy before the samples were taken. In addition, the chemotherapy provided was standardized between patients, which has not changed considerably since then for stage I-III patients, so as to provide us a pretherapeutic metabolic profile. 


\section{Appendix B}

Table A1. Multivariate analyses of the associations between individual metabolites and 5-year OS by sex, using patients with complete information of KRAS mutation, BRAF mutation, and MSI status $(\mathrm{N}=136$, females $=67$, males $=69)$.

\begin{tabular}{|c|c|c|c|c|c|c|c|}
\hline \multirow{2}{*}{ Metabolite Name } & \multicolumn{3}{|c|}{ Females } & \multicolumn{3}{|c|}{ Males } & \multirow{2}{*}{$\begin{array}{l}\text { Int. Sex } p \\
\text { Value }\end{array}$} \\
\hline & HR & $95 \%$ CI & $p$ Value $^{\mathrm{a}}$ & HR & $95 \%$ CI & $p$ Value $^{\mathrm{a}}$ & \\
\hline Acetyl-lysine & 1.06 & $0.76-1.49$ & 0.735 & 0.57 & $0.42-0.78$ & $<0.001$ & 0.025 \\
\hline Adenosine & 1.01 & $0.62-1.63$ & 0.976 & 1.42 & $1.04-1.94$ & 0.029 & 0.254 \\
\hline ADMA & 1.58 & $0.90-2.78$ & 0.109 & 0.51 & $0.33-0.80$ & 0.003 & 0.002 \\
\hline Alanine & 1.43 & $0.89-2.30$ & 0.144 & 0.65 & $0.46-0.91$ & 0.012 & 0.007 \\
\hline Arginine & 1.70 & $0.72-4.03$ & 0.227 & 0.53 & $0.28-0.99$ & 0.045 & 0.049 \\
\hline Argininosuccinic acid & 1.08 & $0.71-1.64$ & 0.713 & 0.58 & $0.40-0.83$ & 0.003 & 0.026 \\
\hline Citrulline & 1.76 & $0.97-3.18$ & 0.063 & 0.44 & $0.25-0.77$ & 0.004 & 0.001 \\
\hline $\mathrm{CMP}$ & 1.27 & $0.55-2.90$ & 0.578 & 0.57 & $0.36-0.90$ & 0.017 & 0.076 \\
\hline Glycerol 3-phosphate & 4.20 & $1.24-14.25$ & 0.021 & 1.03 & $0.39-2.74$ & 0.948 & 0.057 \\
\hline GMP & 1.13 & $0.62-2.07$ & 0.686 & 0.59 & $0.39-0.89$ & 0.013 & 0.055 \\
\hline Histidine & 1.23 & $0.72-2.08$ & 0.451 & 0.73 & $0.53-1.00$ & 0.049 & 0.080 \\
\hline Lysine & 1.54 & $0.63-3.74$ & 0.341 & 0.54 & $0.30-0.98$ & 0.044 & 0.030 \\
\hline LysoPC(16:0) & 2.03 & $1.18-3.49$ & 0.010 & 0.77 & $0.52-1.14$ & 0.188 & 0.003 \\
\hline LysoPC(16:1) & 1.13 & $0.69-1.86$ & 0.632 & 0.67 & $0.45-0.98$ & 0.038 & 0.088 \\
\hline Methionine & 2.38 & $0.81-7.00$ & 0.114 & 0.35 & $0.17-0.75$ & 0.007 & 0.009 \\
\hline Ornithine & 1.63 & $0.59-4.50$ & 0.347 & 0.41 & $0.19-0.91$ & 0.028 & 0.032 \\
\hline Phenylalanine & 1.68 & $0.55-5.08$ & 0.359 & 0.26 & $0.09-0.71$ & 0.008 & 0.027 \\
\hline Serine & 2.88 & $0.82-10.15$ & 0.100 & 0.34 & $0.16-0.71$ & 0.004 & 0.005 \\
\hline Spermine & 2.13 & $1.29-3.52$ & 0.003 & 1.16 & $0.82-1.63$ & 0.407 & 0.076 \\
\hline Taurine & 0.63 & $0.19-2.11$ & 0.451 & 0.34 & $0.14-0.79$ & 0.013 & 0.475 \\
\hline Threonine & 1.38 & $0.62-3.08$ & 0.432 & 0.39 & $0.20-0.75$ & 0.005 & 0.012 \\
\hline Tyrosine & 0.81 & $0.40-1.68$ & 0.578 & 0.60 & $0.37-0.99$ & 0.044 & 0.325 \\
\hline Uracil & 2.87 & $0.70-11.76$ & 0.143 & 0.30 & $0.16-0.58$ & $<0.001$ & 0.005 \\
\hline Xanthosine & 1.21 & $0.85-1.73$ & 0.288 & 0.56 & $0.37-0.84$ & 0.006 & 0.016 \\
\hline
\end{tabular}

${ }^{\mathrm{a}} p$ values for the association between individual metabolites and 5-year OS calculated by multivariate Cox $\mathrm{PH}$ regression models, adjusted for anatomic location, clinical stages, chemotherapy history, age, KRAS mutation, $B R A F$ mutation, and MSI status (before FDR adjustment). The abundance of each metabolite was treated as a continuous variable and was $\log _{2}$ transformed. A metabolite with $\mathrm{HR}<1$ was associated with better OS; a metabolite with HR $>1$ was associated with worse OS. ${ }^{\mathrm{b}} p$ value of the interaction between the metabolite and sex. Int. Sex $p$ value $<0.05$ indicate a significant sex interaction.

Table A2. Multivariate analyses of the associations between individual metabolites and 5-year RFS by sex, using patients with complete information of KRAS mutation, BRAF mutation, and MSI status $(\mathrm{N}=136$, females $=67$, males $=69)$.

\begin{tabular}{|c|c|c|c|c|c|c|c|}
\hline \multirow{2}{*}{ Metabolite Name } & \multicolumn{3}{|c|}{ Females } & \multicolumn{3}{|c|}{ Males } & \multirow{2}{*}{$\begin{array}{l}\text { Int. Sex } p \\
\text { Value }\end{array}$} \\
\hline & HR & $95 \%$ CI & $p$ Value ${ }^{a}$ & HR & $95 \%$ CI & $p$ Value ${ }^{a}$ & \\
\hline Arginine & 3.05 & $1.33-7.02$ & 0.009 & 1.88 & $0.64-5.52$ & 0.252 & 0.218 \\
\hline Asparagine & 2.60 & $1.06-6.37$ & 0.036 & 1.02 & $0.56-1.85$ & 0.943 & 0.058 \\
\hline Glutathione disulfide & 0.67 & $0.47-0.94$ & 0.022 & 0.84 & $0.62-1.15$ & 0.274 & 0.264 \\
\hline Hypoxanthine & 6.02 & $0.55-65.93$ & 0.142 & 0.37 & $0.17-0.80$ & 0.011 & 0.023 \\
\hline LysoPC(16:0) & 1.94 & $1.11-3.37$ & 0.020 & 0.69 & $0.39-1.22$ & 0.202 & 0.025 \\
\hline LysoPE(18:2) & 1.75 & $0.79-3.89$ & 0.167 & 0.40 & $0.17-0.94$ & 0.035 & 0.059 \\
\hline Methionine & 3.40 & $1.10-10.53$ & 0.034 & 1.12 & $0.43-2.89$ & 0.814 & 0.095 \\
\hline N1-acetylspermine & 0.95 & $0.66-1.36$ & 0.781 & 3.20 & $1.32-7.76$ & 0.010 & 0.016 \\
\hline Succinate & 0.87 & $0.25-3.11$ & 0.834 & 0.07 & $0.01-0.92$ & 0.043 & 0.143 \\
\hline
\end{tabular}

${ }^{a} p$ values for the association between individual metabolites and 5-year RFS calculated by multivariate Cox $\mathrm{PH}$ regression models, adjusted for anatomic location, clinical stages, chemotherapy history, age, KRAS mutation, $B R A F$ mutation, and MSI status (before FDR adjustment). The abundance of each metabolite was treated as a continuous variable and was $\log _{2}$ transformed. A metabolite with $\mathrm{HR}<1$ was associated with better RFS; a metabolite with HR $>1$ was associated with worse RFS. ${ }^{b} p$ value of the interaction between the metabolite and sex. Int. Sex $p$ value $<0.05$ indicate a significant sex interaction. 
Table A3. Multivariate analysis of association between metabolites in ASNS-catalyzed asparagine synthesis pathway and OS and RFS for all patients using data with complete records of KRAS mutation information $(\mathrm{N}=161$, females $=78$, males $=83$ ).

\begin{tabular}{|c|c|c|c|c|c|c|c|c|}
\hline \multirow[b]{2}{*}{ Variable $^{a}$} & \multicolumn{4}{|c|}{ OS } & \multicolumn{4}{|c|}{ RFS } \\
\hline & HR & $95 \%$ CI & $p$ Value & $\begin{array}{l}\text { Int. Sex } p \\
\text { Value }^{b}\end{array}$ & HR & $95 \%$ CI & $p$ Value & $\begin{array}{l}\text { Int. Sex } p \\
\text { Value }{ }^{b}\end{array}$ \\
\hline Asparagine & 1.28 & $0.74-2.22$ & 0.377 & 0.035 & 2.50 & $1.27-4.91$ & 0.008 & 0.010 \\
\hline Aspartate & 1.24 & $0.80-1.93$ & 0.345 & 0.967 & 1.59 & $0.96-2.63$ & 0.071 & 0.447 \\
\hline Glutamate & 0.68 & $0.26-1.80$ & 0.438 & 0.692 & 0.43 & $0.13-1.41$ & 0.165 & 0.078 \\
\hline Glutamine & 0.65 & $0.24-1.75$ & 0.393 & 0.932 & 0.21 & $0.07-0.63$ & 0.005 & 0.716 \\
\hline AMP & 0.74 & $0.47-1.17$ & 0.198 & 0.444 & 0.86 & $0.50-1.48$ & 0.585 & 0.319 \\
\hline Sex = Male (ref: female) & 2.02 & $0.94-4.32$ & 0.070 & - & 0.97 & $0.40-2.34$ & 0.947 & - \\
\hline $\begin{array}{c}\text { Anatomic location }=R C C \\
\text { (ref: } L C C)\end{array}$ & 0.81 & $0.37-1.78$ & 0.598 & - & 0.68 & $0.28-1.70$ & 0.415 & - \\
\hline $\begin{array}{c}\text { Clinical stage }=\text { late (ref: } \\
\text { early) }\end{array}$ & 4.46 & $1.55-12.78$ & 0.005 & - & 1.32 & $0.37-4.62$ & 0.669 & - \\
\hline $\begin{array}{c}\text { Chemotherapy }=\text { yes (ref: } \\
\text { no) }\end{array}$ & 1.05 & $0.36-3.10$ & 0.924 & & 2.58 & $0.67-9.96$ & 0.166 & \\
\hline Age & 1.10 & $1.04-1.16$ & $<0.001$ & - & 0.98 & $0.92-1.05$ & 0.604 & - \\
\hline$K R A S=\underset{\text { type })}{\text { mutant }}$ (ref: wild & 1.09 & $0.50-2.37$ & 0.832 & - & 1.48 & $0.60-3.69$ & 0.398 & - \\
\hline
\end{tabular}

${ }^{a}$ All of the variables listed were included in one multivariate Cox PH model, where the abundance of each metabolite was treated as a continuous variable and was $\log _{2}$ transformed. ${ }^{b} p$ value of the interaction between the metabolite and sex.

Table A4. Multivariate analysis of association between metabolites in ASNS-catalyzed asparagine synthesis metabolic pathway and OS and RFS by sex using data with complete records of KRAS mutation information ( $\mathrm{N}=161$, females $=78$, males $=83$ ).

\begin{tabular}{|c|c|c|c|c|c|c|c|c|c|c|c|c|}
\hline \multirow{3}{*}{ Variable $^{a}$} & \multicolumn{6}{|c|}{ os } & \multicolumn{6}{|c|}{ RFS } \\
\hline & \multicolumn{3}{|c|}{ Females } & \multicolumn{3}{|c|}{ Males } & \multicolumn{3}{|c|}{ Females } & \multicolumn{3}{|c|}{ Males } \\
\hline & HR & $95 \%$ CI & $p$ Value & HR & $95 \%$ CI & $p$ Value & HR & $95 \% \mathrm{CI}$ & $p$ Value & HR & $95 \%$ CI & $p$ Value \\
\hline Asparagine & 6.79 & $1.43-32.16$ & 0.016 & 0.77 & $0.42-1.42$ & 0.407 & 15.23 & $\begin{array}{c}2.23- \\
104.27\end{array}$ & 0.006 & 1.37 & $0.66-2.82$ & 0.397 \\
\hline Aspartate & 1.01 & $0.35-2.92$ & 0.984 & 1.33 & $0.76-2.32$ & 0.319 & 1.38 & $0.42-4.49$ & 0.594 & 1.55 & $0.81-2.97$ & 0.190 \\
\hline Glutamate & 0.30 & $0.04-1.99$ & 0.211 & 1.21 & $0.34-4.31$ & 0.771 & 0.32 & $0.03-3.50$ & 0.348 & 0.42 & $0.08-2.12$ & 0.291 \\
\hline Glutamine & 0.12 & $0.01-0.93$ & 0.044 & 0.96 & $0.24-3.74$ & 0.949 & 0.02 & $0.002-0.21$ & 0.001 & 0.39 & $0.07-2.11$ & 0.276 \\
\hline AMP & 0.99 & $0.35-2.77$ & 0.981 & 0.62 & $0.34-1.13$ & 0.117 & 1.07 & $0.33-3.45$ & 0.915 & 0.76 & $0.32-1.88$ & 0.554 \\
\hline $\begin{array}{l}\text { KRAS = mutant } \\
\text { (ref: wild type) }\end{array}$ & 1.34 & $0.32-5.67$ & 0.69 & 0.54 & $0.17-1.68$ & 0.286 & 1.69 & $0.36-7.94$ & 0.505 & 0.77 & $0.16-3.76$ & 0.748 \\
\hline $\begin{array}{l}\text { Anatomic location }=\text { RCC } \\
\text { (ref: LCC) }\end{array}$ & 1.50 & $0.43-5.31$ & 0.527 & 0.83 & $0.26-2.71$ & 0.759 & 0.82 & $0.21-3.25$ & 0.777 & 0.76 & $0.17-3.47$ & 0.721 \\
\hline $\begin{array}{c}\text { Clinical stage = late } \\
\text { (ref: early) }\end{array}$ & 9.66 & $1.34-69.57$ & 0.024 & 8.09 & $1.42-46.22$ & 0.019 & 1.31 & $0.19-9.03$ & 0.782 & 0.61 & $0.07-4.78$ & 0.636 \\
\hline $\begin{array}{c}\text { Chemotherapy }=\text { Yes } \\
\text { (ref: no) }\end{array}$ & 1.80 & $0.37-8.74$ & 0.468 & 0.46 & $0.07-2.85$ & 0.402 & 3.55 & $0.47-26.49$ & 0.217 & 3.33 & $0.37-29.78$ & 0.283 \\
\hline Age & 1.09 & $0.97-1.23$ & 0.154 & 1.14 & $1.05-1.231$ & 0.001 & 1.05 & $0.94-1.17$ & 0.407 & 0.95 & $0.87-1.05$ & 0.331 \\
\hline
\end{tabular}

${ }^{a}$ All of the variables listed were included in one multivariate Cox PH model. The abundance of each metabolite was treated as a continuous variable and was $\log _{2}$ transformed.

\section{References}

1. Colorectal Cancer Statistics. Available online: https://www.cdc.gov/cancer/colorectal/statistics/index.htm (accessed on 13 October 2021).

2. Amin, M.B.; Edge, S.B. AJCC Cancer Staging Manual; Springer: Berlin, Germany, 2017.

3. TNM Classification of Malignant Tumours. Available online: https://www.uicc.org/resources/tnm (accessed on 13 October 2021).

4. Nakagawa-Senda, H.; Hori, M.; Matsuda, T.; Ito, H. Prognostic Impact of Tumor Location in Colon Cancer: The Monitoring of Cancer Incidence in Japan (MCIJ) Project. BMC Cancer 2019, 19, 431. [CrossRef] [PubMed]

5. Zheng, C.; Jiang, F.; Lin, H.; Li, S. Clinical Characteristics and Prognosis of Different Primary Tumor Location in Colorectal Cancer: A Population-Based Cohort Study. Clin. Transl. Oncol. 2019, 21, 1524-1531. [CrossRef] [PubMed]

6. Doleman, B.; Mills, K.T.; Lim, S.; Zelhart, M.D.; Gagliardi, G. Body Mass Index and Colorectal Cancer Prognosis: A Systematic Review and Meta-Analysis. Tech. Coloproctol. 2016, 20, 517-535. [CrossRef] [PubMed] 
7. Zhu, B.; Wu, X.; Wu, B.; Pei, D.; Zhang, L.; Wei, L. The Relationship between Diabetes and Colorectal Cancer Prognosis: A Meta-Analysis Based on the Cohort Studies. PLoS ONE 2017, 12, e0176068. [CrossRef]

8. Xu, G.; Zhang, M.; Zhu, H.; Xu, J. A 15-Gene Signature for Prediction of Colon Cancer Recurrence and Prognosis Based on SVM. Gene 2017, 604, 33-40. [CrossRef]

9. Pietrzyk, Ł.; Korolczuk, A.; Matysek, M.; Arciszewski, M.B.; Torres, K. Clinical Value of Detecting Tumor Endothelial Marker 8 (ANTXR1) as a Biomarker in the Diagnosis and Prognosis of Colorectal Cancer. Cancer Manag. Res. 2021, 13, 3113-3122. [CrossRef]

10. Peng, Q.; Zhang, X.; Min, M.; Zou, L.; Shen, P.; Zhu, Y. The Clinical Role of Microrna-21 as a Promising Biomarker in the Diagnosis and Prognosis of Colorectal Cancer: A Systematic Review and Meta-Analysis. Oncotarget 2017, 8, 44893-44909. [CrossRef]

11. He, T.; Zhang, M.; Zheng, R.; Zheng, S.; Linghu, E.; Herman, J.G.; Guo, M. Methylation of SLFN11 Is a Marker of Poor Prognosis and Cisplatin Resistance in Colorectal Cancer. Epigenomics 2017, 9, 849-862. [CrossRef]

12. Hsu, C.-H.; Hsiao, C.-W.; Sun, C.-A.; Wu, W.-C.; Yang, T.; Hu, J.-M.; Liao, Y.-C.; Huang, C.-H.; Chen, C.-Y.; Lin, F.-H.; et al Multiple Gene Promoter Methylation and Clinical Stage in Adjacent Normal Tissues: Effect on Prognosis of Colorectal Cancer in Taiwan. Sci. Rep. 2020, 10, 145. [CrossRef] [PubMed]

13. Schmidt, D.R.; Patel, R.; Kirsch, D.G.; Lewis, C.A.; Vander Heiden, M.G.; Locasale, J.W. Metabolomics in Cancer Research and Emerging Applications in Clinical Oncology. CA Cancer J. Clin. 2021, 71, 333-358. [CrossRef]

14. Zhang, F.; Zhang, Y.; Zhao, W.; Deng, K.; Wang, Z.; Yang, C.; Ma, L.; Openkova, M.S.; Hou, Y.; Li, K. Metabolomics for Biomarker Discovery in the Diagnosis, Prognosis, Survival and Recurrence of Colorectal Cancer: A Systematic Review. Oncotarget 2017, 8, 35460-35472. [CrossRef] [PubMed]

15. Ose, J.; Gigic, B.; Brezina, S.; Lin, T.; Baierl, A.; Geijsen, A.J.; van Roekel, E.; Robinot, N.; Gicquiau, A.; Achaintre, D.; et al. Targeted plasma metabolic profiles and risk of recurrence in Stage ii and III Colorectal Cancer Patients: Results from an International Cohort Consortium. Metabolites 2021, 11, 129. [CrossRef] [PubMed]

16. Berger, M.D.; Yang, D.; Sunakawa, Y.; Zhang, W.; Ning, Y.; Matsusaka, S.; Okazaki, S.; Miyamoto, Y.; Suenaga, M.; Schirripa, M.; et al. Impact of Sex, Age, and Ethnicity/Race on the Survival of Patients with Rectal Cancer in the United States from 1988 to 2012. Oncotarget 2016, 7, 53668-53678. [CrossRef] [PubMed]

17. Abancens, M.; Bustos, V.; Harvey, H.; McBryan, J.; Harvey, B.J. Sexual Dimorphism in Colon Cancer. Front. Oncol. 2020, 10, 607909. [CrossRef] [PubMed]

18. Petrelli, F.; Tomasello, G.; Borgonovo, K.; Ghidini, M.; Turati, L.; Dallera, P.; Passalacqua, R.; Sgroi, G.; Barni, S. Prognostic Survival Associated with Left-Sided vs Right-Sided Colon Cancer. JAMA Oncol. 2017, 3, 211. [CrossRef]

19. Hansen, I.O.; Jess, P. Possible better long-term survival in left versus right-sided colon cancer-A systematic review. Dan. Med. J. 2012, 59, A4444

20. Majek, O.; Gondos, A.; Jansen, L.; Emrich, K.; Holleczek, B.; Katalinic, A.; Nennecke, A.; Eberle, A.; Brenner, H. Sex Differences in Colorectal Cancer Survival: Population-Based Analysis of 164,996 Colorectal Cancer Patients in Germany. PLoS ONE 2013, 8, e68077. [CrossRef]

21. Cai, Y.; Rattray, N.J.; Zhang, Q.; Mironova, V.; Santos-Neto, A.; Hsu, K.-S.; Rattray, Z.; Cross, J.R.; Zhang, Y.; Paty, P.B.; et al. Sex Differences in Colon Cancer Metabolism Reveal a Novel Subphenotype. Sci. Rep. 2020, 10, 4905. [CrossRef]

22. Richards, N.G.; Kilberg, M.S. Asparagine Synthetase Chemotherapy. Annu. Rev. Biochem. 2006, 75, 629-654. [CrossRef]

23. Zhang, J.; Fan, J.; Venneti, S.; Cross, J.R.; Takagi, T.; Bhinder, B.; Djaballah, H.; Kanai, M.; Cheng, E.H.; Judkins, A.R.; et al. Asparagine Plays a Critical Role in Regulating Cellular Adaptation to Glutamine Depletion. Mol. Cell 2014, 56, 205-218. [CrossRef]

24. Lin, H.H.; Chung, Y.; Cheng, C.-T.; Ouyang, C.; Fu, Y.; Kuo, C.-Y.; Chi, K.K.; Sadeghi, M.; Chu, P.; Kung, H.-J.; et al. Autophagic Reliance Promotes Metabolic Reprogramming in Oncogenic Kras-Driven Tumorigenesis. Autophagy 2018, 14, 1481-1498. [CrossRef] [PubMed]

25. Krall, A.S.; Xu, S.; Graeber, T.G.; Braas, D.; Christofk, H.R. Asparagine Promotes Cancer Cell Proliferation through Use as an Amino Acid Exchange Factor. Nat. Commun. 2016, 7, 11457. [CrossRef] [PubMed]

26. Knott, S.R.; Wagenblast, E.; Khan, S.; Kim, S.Y.; Soto, M.; Wagner, M.; Turgeon, M.-O.; Fish, L.; Erard, N.; Gable, A.L.; et al Asparagine bioavailability governs metastasis in a model of breast cancer. Nature 2018, 554, 378-381. [CrossRef]

27. Du, F.; Chen, J.; Liu, H.; Cai, Y.; Cao, T.; Han, W.; Yi, X.; Qian, M.; Tian, D.; Nie, Y.; et al. Sox12 promotes colorectal cancer cell proliferation and metastasis by regulating asparagine synthesis. Cell Death Dis. 2019, 10, 239. [CrossRef] [PubMed]

28. Toda, K.; Kawada, K.; Iwamoto, M.; Inamoto, S.; Sasazuki, T.; Shirasawa, S.; Hasegawa, S.; Sakai, Y. Metabolic alterations caused by KRAS mutations in colorectal cancer contribute to cell adaptation to glutamine depletion by upregulation of asparagine synthetase. Neoplasia 2016, 18, 654-665. [CrossRef] [PubMed]

29. Gwinn, D.M.; Lee, A.G.; Briones-Martin-del-Campo, M.; Conn, C.S.; Simpson, D.R.; Scott, A.I.; Le, A.; Cowan, T.M.; Ruggero, D.; Sweet-Cordero, E.A. Oncogenic kras regulates amino acid homeostasis and asparagine biosynthesis via ATF4 and alters sensitivity to L-asparaginase. Cancer Cell 2018, 33, 91-107. [CrossRef]

30. Wong, C.C.; Qian, Y.; Li, X.; Xu, J.; Kang, W.; Tong, J.H.; To, K.-F.; Jin, Y.; Li, W.; Chen, H.; et al. SLC25A22 promotes proliferation and survival of colorectal cancer cells with KRAS mutations and xenograft tumor progression in mice via intracellular synthesis of aspartate. Gastroenterology 2016, 151, 945-960. [CrossRef] [PubMed]

31. Li, X.; Chung, A.C.K.; Li, S.; Wu, L.; Xu, J.; Yu, J.; Wong, C.; Cai, Z. LC-MS-based metabolomics revealed slc25a22 as an essential regulator of aspartate-derived amino acids and polyamines in KRAS-mutant colorectal cancer. Oncotarget 2017, 8, 101333-101344. [CrossRef] 
32. Hinze, L.; Labrosse, R.; Degar, J.; Han, T.; Schatoff, E.M.; Schreek, S.; Karim, S.; McGuckin, C.; Sacher, J.R.; Wagner, F.; et al. Exploiting the therapeutic interaction of Wnt pathway activation and asparaginase for colorectal cancer therapy. Cancer Discov. 2020, 10, 1690-1705. [CrossRef]

33. Chiu, M.; Taurino, G.; Bianchi, M.G.; Kilberg, M.S.; Bussolati, O. Asparagine synthetase in Cancer: Beyond acute lymphoblastic leukemia. Front. Oncol. 2020, 9, 1480. [CrossRef]

34. Montrose, D.C.; Saha, S.; Foronda, M.; McNally, E.M.; Chen, J.; Zhou, X.K.; Ha, T.; Krumsiek, J.; Buyukozkan, M.; Verma, A.; et al. Exogenous and endogenous sources of serine contribute to colon cancer metabolism, growth, and resistance to 5-Fluorouracil. Cancer Res. 2021, 81, 2275-2288. [CrossRef] [PubMed]

35. Grant, G.A. D-3-phosphoglycerate dehydrogenase. Front. Mol. Biosci. 2018, 5, 110. [CrossRef] [PubMed]

36. Yoon, S.; Kim, J.G.; Seo, A.N.; Park, S.Y.; Kim, H.J.; Park, J.S.; Choi, G.S.; Jeong, J.Y.; Jun, D.Y.; Yoon, G.S.; et al. Clinical implication of serine metabolism-associated enzymes in colon cancer. Oncology 2015, 89, 351-359. [CrossRef]

37. Bednarz-Misa, I.; Fleszar, M.G.; Zawadzki, M.; Kapturkiewicz, B.; Kubiak, A.; Neubauer, K.; Witkiewicz, W.; Krzystek-Korpacka, M. L-arginine/no pathway metabolites in colorectal cancer: Relevance as disease biomarkers and predictors of adverse clinical outcomes following surgery. J. Clin. Med. 2020, 9, 1782. [CrossRef] [PubMed]

38. Curis, E.; Nicolis, I.; Moinard, C.; Osowska, S.; Zerrouk, N.; Bénazeth, S.; Cynober, L. Almost all about citrulline in mammals. Amino Acids 2005, 29, 177-205. [CrossRef] [PubMed]

39. Pegg, A.E. Regulation of ornithine decarboxylase. J. Biol. Chem. 2006, 281, 14529-14532. [CrossRef]

40. Laukaitis, C.M.; Gerner, E.W. DFMO: Targeted risk reduction therapy for colorectal neoplasia. Best Pract. Res. Clin. Gastroenterol. 2011, 25, 495-506. [CrossRef]

41. S0820, Adenoma and Second Primary Prevention Trial. Available online: https://clinicaltrials.gov/ct2/show /NCT01349881 (accessed on 13 October 2021).

42. Wanders, D.; Hobson, K.; Ji, X. Methionine restriction and Cancer Biology. Nutrients 2020, 12, 684. [CrossRef]

43. Ruoppolo, M.; Scolamiero, E.; Caterino, M.; Mirisola, V.; Franconi, F.; Campesi, I. Female and male human babies have distinct blood metabolomic patterns. Mol. Biosyst. 2015, 11, 2483-2492. [CrossRef]

44. Bell, J.A.; Santos Ferreira, D.L.; Fraser, A.; Soares, A.L.; Howe, L.D.; Lawlor, D.A.; Carslake, D.; Davey Smith, G.; O'Keeffe, L.M. Sex differences in systemic metabolites at four life stages: Cohort Study with repeated metabolomics. BMC Med. 2021, 19, 58. [CrossRef]

45. Clocchiatti, A.; Cora, E.; Zhang, Y.; Dotto, G.P. Sexual dimorphism in cancer. Nat. Rev. Cancer 2016, 16, 330-339. [CrossRef] [PubMed]

46. Kovács, T.; Szabó-Meleg, E.; Ábrahám, I.M. Estradiol-induced epigenetically mediated mechanisms and regulation of gene expression. Int. J. Mol. Sci. 2020, 21, 3177. [CrossRef] [PubMed]

47. Kim, Y.S.; Unno, T.; Kim, B.-Y.; Park, M.-S. Sex differences in gut microbiota. World J. Men's Health 2020, 38, 48-60. [CrossRef] [PubMed]

48. Zhu, G.; Pei, L.; Xia, H.; Tang, Q.; Bi, F. Role of oncogenic kras in the prognosis, diagnosis and treatment of colorectal cancer. Mol. Cancer 2021, 20, 143. [CrossRef] [PubMed]

49. Sanz-Garcia, E.; Argiles, G.; Elez, E.; Tabernero, J. BRAF mutant colorectal cancer: Prognosis, treatment, and New Perspectives. Ann. Oncol. 2017, 28, 2648-2657. [CrossRef]

50. Popat, S.; Hubner, R.; Houlston, R.S. Systematic review of microsatellite instability and colorectal cancer prognosis. J. Clin. Oncol. 2005, 23, 609-618. [CrossRef] 